\title{
Differentiation of bone marrow-derived mesenchymal stem cells into chondrocytes using chondrocyte extract
}

\author{
XINJIE TANG ${ }^{*}$, LINGLING SHENG ${ }^{*}$, FENG XIE and QUN ZHANG \\ Department of Plastic and Reconstructive Surgery, Shanghai Ninth People's Hospital, \\ Shanghai Jiao Tong University, School of Medicine, Shanghai 200011, P.R. China
}

Received February 28, 2012; Accepted June 29, 2012

DOI: $110.3892 / \mathrm{mmr} .2012 .996$

\begin{abstract}
Reprogramming cells using cell extracts is an effective method for harvesting cells of interest. The aim of this study was to investigate the chondrogenic transdifferentiation potential of swine bone marrow-derived mesenchymal stem cells (BM-MSCs) by culturing with chondrocyte extract, using monolayer and micromass culture. Chondrogenic-specific markers were detected via reverse transcription-polymerase chain reaction (RT-PCR) and immunofluorescence. After 7 days of induction in monolayer culture, BM-MSCs reversibly permeabilized with streptolysin O (SLO), a bacterial exotoxin that is capable of forming large pores in the plasma membrane of mammalian cells, and expressed chondrocyte-specific genes such as type II collagen (COL II) and aggrecan. A positive protein expression of COL II was also observed. However, BM-MSCs treated without SLO did not express the related genes and proteins. The transition of reprogrammed BM-MSCs was lost 14 days later. By using micromass culture, reprogrammed BM-MSCs were able to maintain the change until the 14th day. In summary, permeabilized BM-MSCs were transiently transdifferentiated into chondrocytes by co-culturing with the chondrocyte extract. Moreover, a highdensity culture method was able to increase the time in which the phenotypic change was maintained.
\end{abstract}

\section{Introduction}

Transplantation of autogenous or allogenous chondrocytes has been used to repair defective cartilage. However, the limited

Correspondence to: Dr Qun Zhang, Department of Plastic and Reconstructive Surgery, Shanghai Ninth People's Hospital, Shanghai Jiao Tong University, School of Medicine, 639 Zhizaoju Road, Shanghai 200011, P.R. China

E-mail: zhangqun_639@163.com

${ }^{*}$ Contributed equally

Key words: bone marrow-derived mesenchymal stem cells, cell extract, chondrocytes, reprogramming, streptolysin $\mathrm{O}$ sources for a sufficient number of chondrocytes restricts the development and clinical application of chondrocyte-based therapies (1). Therefore, finding an effective method to collect 'target cells' is crucial.

Increasing evidence has shown that an extract harvested from one type of differentiated somatic cell is capable of altering another cell phenotype in vitro, a method of reprogramming that has developed in recent years (2-5). This method was first introduced by Hakelien et al (2). Those authors found that 293T cells permeabilized with streptolysin O (SLO) exposed to a T-cell extract were capable of adopting $\mathrm{T}$ cell-specific properties, such as expressing $\mathrm{T}$ cell-specific receptors and assembling the interleukin-2 receptor. Supported by a number of studies, use of this method suggests that altering cell fate using cell extract is feasible and effective, and may be applied to multiple cell types.

The most common cells used as 'donor cells', which receive another cell extract and differentiate into 'target cells', are fibroblasts and adult stem cells, as they can be harvested easily and exhibit marked proliferative capacity. Compared with fibroblasts, adult stem cells have a broad prospect of application as a result of their potential of multi-directional differentiation into cells including adipocytes, osteoblasts and chondrocytes in response to lineage-specific induction factors $(6,7)$. Bone marrow-derived mesenchymal stem cells (BM-MSCs), widely used in regenerative medicine, have also been successfully induced into chondrocytes by the induction of growth factors $(8,9)$, co-culturing with chondrocytes $(10,11)$ or low-intensity ultrasound (12).

The aim of this study was to observe whether chondrocyte extract induced the differentiation of BM-MSCs into chondrocytes in a monolayer culture. Moreover, we investigated whether the maintenance time of phenotypic alteration could be prolonged and whether cells were able to produce cartilage-specific extracellular matrix (ECM) components when cultured at a high density.

\section{Materials and methods}

Animals. All animal procedures in this study were approved by Shanghai Jiaotong University Medical Center, Institutional Animal Care and Use Committee. The animals were maintained in a specific pathogen-free environment. The surgical procedures were performed under aseptic conditions. 
BM-MSC preparation. Swine bone marrow was obtained from the posterior superior iliac crest of newborn pigs. Briefly, after washing twice in phosphate-buffered saline (PBS; Gibco-BRL, Grand Island, NY, USA) to remove red cell fragments, cells were resuspended in Dulbecco's modified Eagle's medium (DMEM; Gibco) with low glucose plus $10 \%$ fetal bovine serum (FBS; Gibco) and plated in $100 \mathrm{~mm}^{2}$ culture dishes at a density of $2.0 \times 10^{5}$ cells $/ \mathrm{cm}^{2}$ at $37^{\circ} \mathrm{C}$ with $5 \% \mathrm{CO}_{2}$. Non-adherent cells were removed by medium change after 5 days. The adherent cells were collected when $>80 \%$ confluence was achieved and then subcultured. BM-MSCs at passage 2 were used for subsequent experiments.

Chondrocyte isolation and culture. The swine articular cartilage was cut into $2 \times 2 \mathrm{~mm}$ slices and washed twice with PBS. After being digested with $0.25 \%$ trypsin plus $0.02 \%$ EDTA at $37^{\circ} \mathrm{C}$ for $30 \mathrm{~min}$, the cartilage slices were further digested with $0.1 \%$ collagenase II in serum-free DMEM at $37^{\circ} \mathrm{C}$ for $12-16 \mathrm{~h}$. Chondrocytes were then harvested, counted and seeded into culture dishes at a cell density of $2.5 \times 10^{4} / \mathrm{cm}^{2}$ for the culture and subculture in DMEM with high glucose and 10\% FBS. Chondrocytes at passage 1 were used in this study.

Chondrocyte extract. After being harvested, chondrocytes at passage 1 were washed in $\mathrm{Ca}^{2+}$ - and $\mathrm{Mg}^{2+}$-free Hank's balanced salt solution (HBSS, Sigma, St. Louis, MO, USA) twice and cell lysis buffer (100 mM HEPES, pH 8.2, $50 \mathrm{mM} \mathrm{NaCl}$, $5 \mathrm{mM} \mathrm{MgCl}, 1 \mathrm{mM}$ dithiothreitol and protease inhibitors), sedimented at $1,000 \mathrm{rpm}$, resuspended in $0.8 \mathrm{ml}$ cold cell lysis buffer and incubated for $50 \mathrm{~min}$ on ice. Cells were sonicated on ice in $200 \mu \mathrm{l}$ aliquots using a sonicator fitted with a $3-\mathrm{mm}$ diameter probe until the cells and nuclei were lysed, as judged by microscopy. The lysate was sedimented at $15,000 \mathrm{rpm}$ for $15 \mathrm{~min}$ at $4^{\circ} \mathrm{C}$ to pellet the coarse material. The supernatant was aliquoted, frozen in liquid nitrogen and stored at $-80^{\circ} \mathrm{C}$. The protein concentration of the extract was determined by the Bradford assay. In this study, the chondrocyte extract contained $10-15 \mathrm{mg} / \mathrm{ml}$ protein.

Streptolysin $O$-mediated permeabilization and cell extract treatment. BM-MSCs were washed in cold PBS and in cold HBSS. Cells were resuspended in SLO solution at a concentration of 100 cells $/ \mu 1$ with a SLO concentration of $230 \mathrm{ng} / \mathrm{ml}$. Samples were incubated in an $\mathrm{H}_{2} \mathrm{O}$ bath for $50 \mathrm{~min}$ at $37^{\circ} \mathrm{C}$ with occasional agitation. Cells were then collected by sedimentation at $1,800 \mathrm{rpm}$ for $5 \mathrm{~min}$ at $37^{\circ} \mathrm{C}$. A permeabilization efficiency of $>60 \%$ was obtained, as assessed by monitoring the uptake of propidium iodide (PI).

Following permeabilization, cells were resuspended at 1000 cells $/ \mu \mathrm{l}$ in $100 \mu \mathrm{l}$ cell extract containing an ATP- regenerating system and $1 \mathrm{mmol} / \mathrm{l}$ of each NTP. Cells were cultured for $1 \mathrm{~h}$ at $37^{\circ} \mathrm{C}$ with occasional agitation. To reseal the cells, cell suspension with cell extract was diluted with DMEM containing $2 \mathrm{mM} \mathrm{CaCl}_{2}$ and cultured for $2 \mathrm{~h}$ at $37^{\circ} \mathrm{C}$. The supernatant was discarded, fresh DMEM containing no $\mathrm{CaCl}_{2}$ was added and cells were cultured until use. According to the different treatments, there were 4 groups: SLO treatment $(+)$ cell extract(+) group; SLO treatment(-) cell extract(+) group; SLO treatment(-) cell extract(-) group; and chondrocyte group, which was regarded as a positive control.
Immunofluorescence. Immunofluorescence analysis was performed on the seventh day. The cells were fixed with $4 \%$ paraformaldehyde for $15 \mathrm{~min}$. The fixed specimens were washed and incubated with primary anti-collagen type II (COL II) monoclonal antibody (Santa Cruz Biotechnology, Santa Cruz, CA, USA) diluted in $0.5 \%$ bovine serum albumin (BSA) at $4^{\circ} \mathrm{C}$ overnight and then washed and incubated with secondary antibody for $30 \mathrm{~min}$ at $37^{\circ} \mathrm{C}$. DAPI was used as a nuclear counterstain. Images were captured on a NIKON microscope using IPP software.

Transcription analysis. Reverse transcription-polymerase chain reaction (RT-PCR) was performed on the seventh day using the primers: COL II: forward (5'-3'): GATGGGCA GAGGTATAATGAT and reverse (5'-3'): CTTTTTCACC TTTGTCACCAC (product size, $322 \mathrm{bp}$ ); aggrecan: forward (5'-3'): ATGACTCTGGGATCTATCGCT and reverse (5'-3'): CGTAGGTCTCATTGGTGTCA-3 (product size, 352 bp); GADPH: forward (5'-3'): ACATCAAGGAGAAGCTCTGC TACG and reverse (5'-3'): GAGGGGCGATGATCTTGAT CTTCA (product size, $366 \mathrm{bp}$ ). The reactions were performed in a T3 thermocycler (Biometra, Goettingen, Germany). The amplified products were separated on $1.5 \%$ agarose gels and visualized by ethidium bromide (Sigma-Aldrich).

\section{Chondrogenic differentiation of treated-BM-MSCs in micro-} mass culture. Based on the results obtained above, micromass culture was performed. Briefly, reversibly permeabilized BM-MSCs with added cell extract were collected by resuspension at a density of $2.0 \times 10^{7}$ cells $/ \mathrm{ml}$ in the growth medium. The cell suspension was then spotted in $15 \mu \mathrm{l}$ drops into dishes. The cells were incubated for $2 \mathrm{~h}$ at $37^{\circ} \mathrm{C}$ to allow attachment and then maintained in the complete medium until day 14 . The specimens were then embedded in paraffin and cut into $10 \mu \mathrm{m}$ sections. The sections were stained with hematoxylin and eosin (H\&E) and toluidine blue to reveal the histological structure and chondroitin sulfate deposition, which is a substance in cartilage matrix, respectively. The expression of COL II was detected using mouse anti-pig monoclonal antibody (1:100 in 0.5\% BSA, Santa Cruz Biotechnology), followed by horseradish peroxidase (HRP)-conjugated anti-mouse antibody (1:500 in PBS, Santa Cruz Biotechnology) and color development with diaminobenzidine tetrahydrochloride (DAB, Santa Cruz Biotechnology).

\section{Results}

Reversible permeabilization of BM-MSCs. In this study, BM-MSCs were treated with various concentrations of SLO (230, 300 and $400 \mathrm{ng} / \mathrm{ml}$, respectively) and the proliferative ability of cells was detected. Results showed that an enhanced SLO concentration slightly increased permeabilization, however, a marked decrease was observed in the proliferative ability of cells (data not shown). SLO (230 ng/ml) was used, as a permeabilization efficiency of $>55 \%$ was obtained and it was proven to be least detrimental (Fig. 1).

Exposure of BM-MSCs to the chondrocyte extract leads to the induction of chondrocyte-specific genes and protein. Chondrocytes were sonicated and observed under a microscope, which revealed that there was no cell and 
A

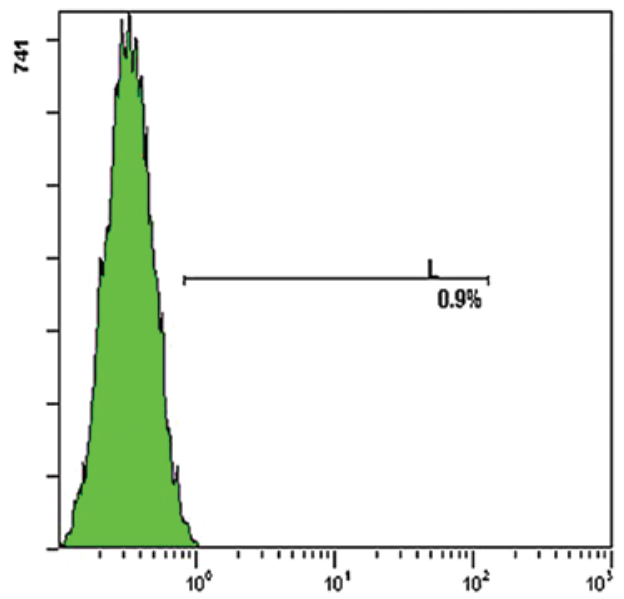

B

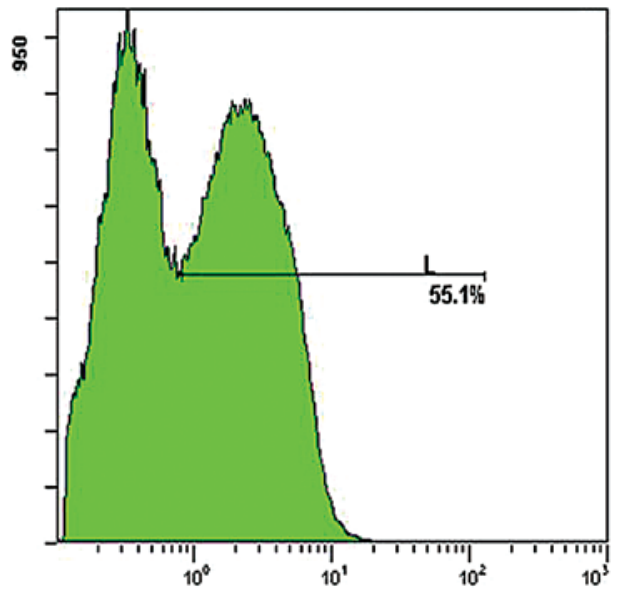

Figure 1. The BM-MSC permeability change after being exposed to SLO. (A) The permeability rate of BM-MSCs without being exposed to SLO. (B) Approximately 55\% BM-MSCs were permeabilized with $230 \mathrm{ng} / \mathrm{ml}$ SLO. BM-MSCs, bone marrow-derived mesenchymal stem cells; SLO, streptolysin $\mathrm{O}$.

nucleus structure (Fig. 2). Consistent with the uptake of PI, following exposure of BM-MSCs to the chondrocyte extract in an ATP-regeneration 7 days later, cells treated with SLO-expressed chondrocyte-specific genes such as COL II and aggrecan on the seventh day (Fig. 3). The immunofluorescent staining revealed that reprogrammed BM-MSCs were positive for COL II (Fig. 4); whereas, no changes were observed in intact (non-permeabilized) BM-MSCs. However, at 14 days, there was no relevant expression.

Maintenance of the chondrogenic phenotype of reprogrammed $B M-M S C s$ in micromass culture. Previous investigations have demonstrated that a high density culture manner, such as micromass or pellet culture, is essential for the chondrogenic differentiation of BM-MSCs. Using monolayer culture, examinations could not detect relevant chondrocyte-specific expression 14 days after cell treatment. Thus, to maintain the chondrogenic differentiation of reprogrammed BM-MSCs, micromass culture was used in this study. When the induction time was extended to 14 days, the cells in the cell mass formed a lacunae structure with chondrocyte-like cells located inside secreting chondroitin sulfate. The expression of COL II was also observed in the cell mass (Fig. 5).
A

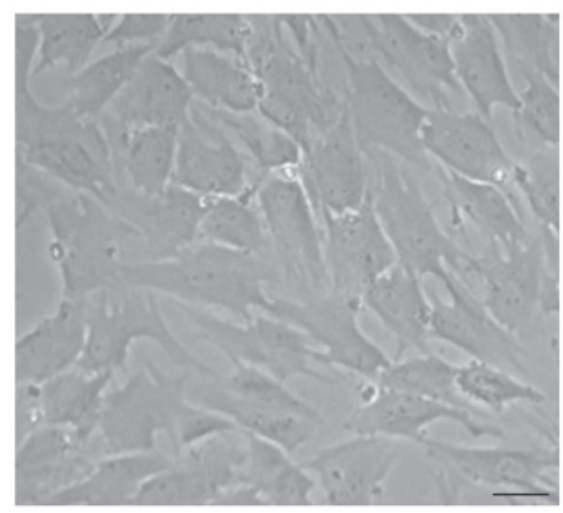

B

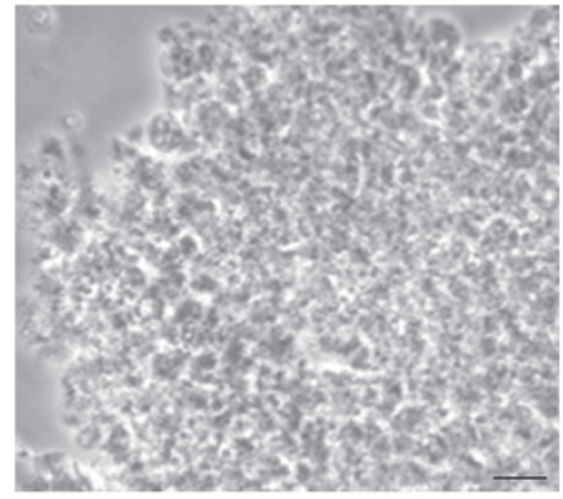

Figure 2. Morphological changes of cells mashed by an ultrasonic processor. (A) Normal chondrocytes and (B) cells mashed by an ultrasonic processor showed no structure (original magnification, $\mathrm{x} 200$ ).

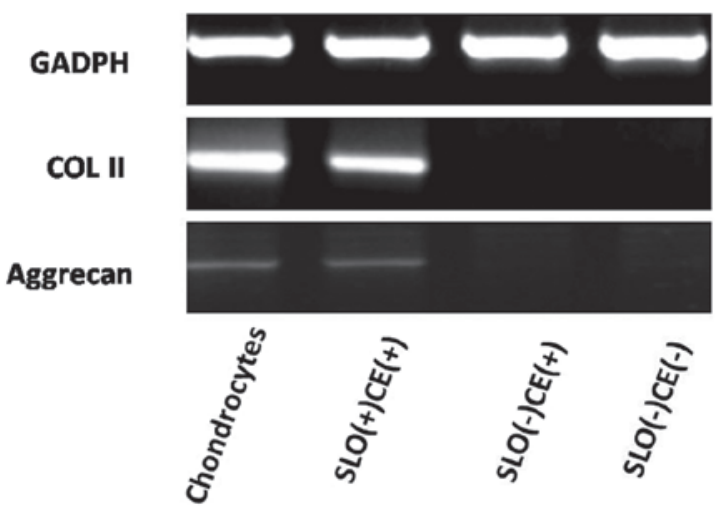

Figure 3. Gene expression of COL II and aggrecan 7 days after cell treatment. COL II, type II collagen.

\section{Discussion}

Cell extract-based systems for reprogramming cell fate have been developed with the aim of eliciting somatic cell transdifferentiation or dedifferentiation. Previous studies have demonstrated that cell extracts harvested from embryonic stem cells (ESCs) (13) or somatic cells (3) are capable of inducing nuclear de-/transdifferentiation of 'donor cells'. The current study provided permeabilized BM-MSCs with the chondrocyte extract and observed changes in the gene and protein expression following cell culture. A high-density culture method was 


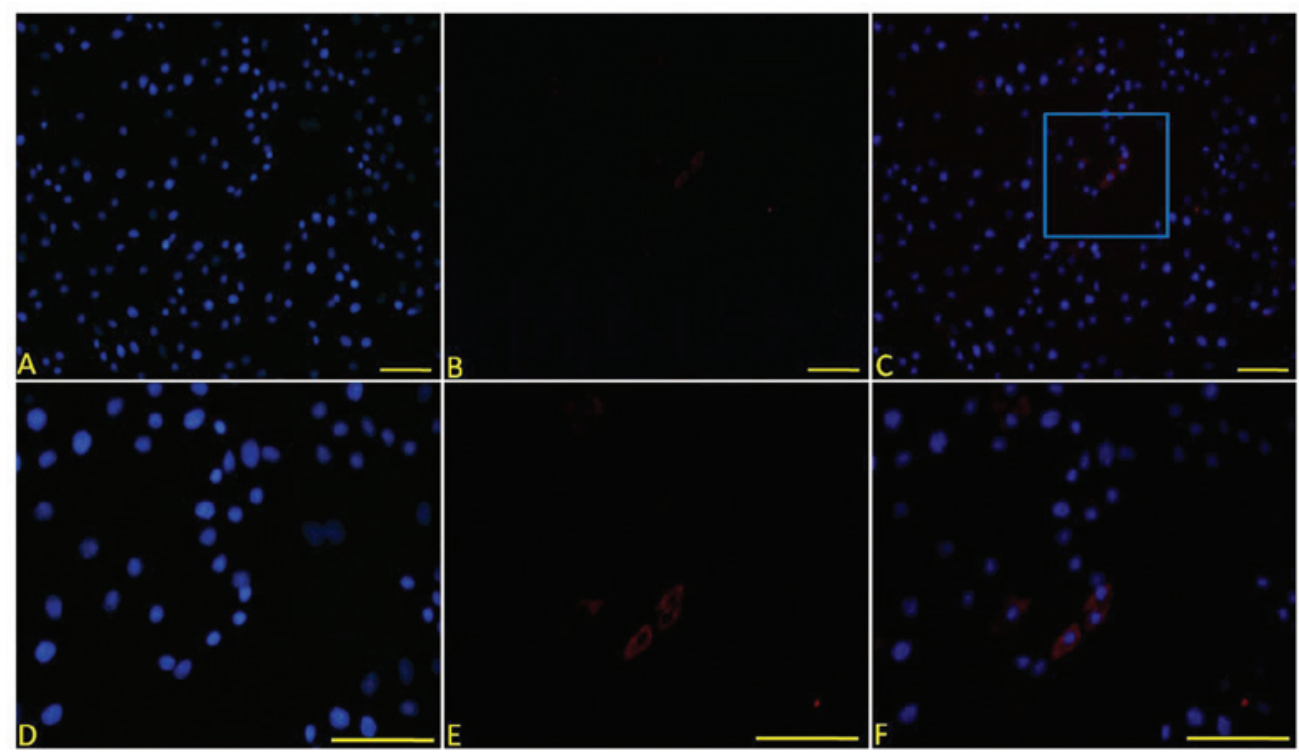

Figure 4. COL II expression in the cells 7 days after cell treatment. COL II was positive in the cells treated with SLO and the cell extract. (A) DAPI staining, (B) anti-COL II immunofluorescent staining, (C) merged image of A and B revealed that some cells expressed COL II, (D) high magnification of DAPI staining of positive cells in the blue frame in (C), (E) high magnification of anti-COL II immunofluorescent staining of positive cells in the blue frame in (C) and (F) merged image of (D) and (E) (original magnification: A-C, x100, D-E, x200). COL II, type II collagen, SLO, streptolysin O.

A

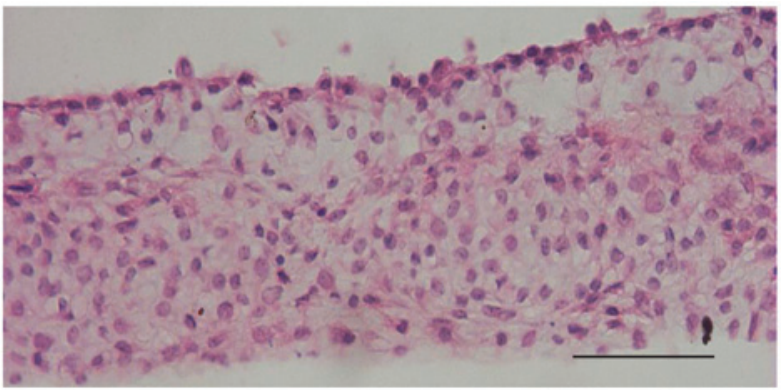

B

C

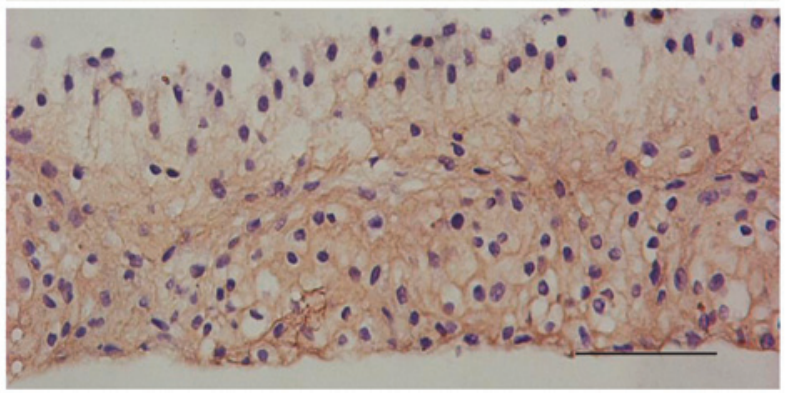

Figure 5. Histological morphology of chondrogenically transdifferentiated BM-MSCs cultured in micromass in the presence of SLO and chondrocyte extract simultaneously on the 14 th day. (A) H\&E staining showed that certain cells in the micromass formed lacunae structure, (B) toluidine blue staining indicated that certain cells secreted chondroitin sulfate, (C) anti-COL II immunohistochemical staining was positive in the cell mass (original magnification, x200). BM-MSCs, bone marrow-derived mesenchymal stem cells, SLO, streptolysin O, COL II, type II collagen, H\&E, hematoxylin and eosin. also found to prolong the maintenance time of the phenotype change of reprogrammed cells.

The main component of the cell extract was thought to be protein, as heat-treated $\left(95^{\circ} \mathrm{C}, 5 \mathrm{~min}\right)$ extracts were not able to induce any changes $(3,5)$. In this study, the cell extract was obtained using cell lysis and ultrasonic homogenization. By this method, the protein molecular weight varies within a wide range. To enable the cell extract to enter 'donor cells' effectively, BM-MSCs were treated with SLO as described previously $(2,3)$. Following the binding of SLO to membranes, toxin monomers diffuse laterally in the bilayer and oligomerize to form homotypic aggregates that represent large transmembrane pores whose diameters may reach $35 \mathrm{~nm}$, allowing molecules $<100 \mathrm{kDa}$ to enter the cell cytosol (14-16). In the presence of $\mathrm{Ca}^{2+}$, the permeabilized cells were resealed for subsequent culture. This finding was consistent with other authors who reported that resealing was temperatureindependent and even occurred eat $4^{\circ} \mathrm{C}(14)$.

Findings of previous studies have shown that a rapid gene change may be induced over a 1-8 $\mathrm{h}$ culture (17) and that these genes would consistently persist up to days $7-10(3,18)$. In our study, no gene alteration was evident on day 3. However, 7 days later, a clear expression of COL II and aggrecan, the two main chondrogenic gene markers, was demonstrated by RT-PCR analysis in the group of permeabilized BM-MSCs in the presence of the cell extract. Consistent with gene change, COL II protein expression was also positive in this group. These results suggested that exposure to the chondrocyte extract initiated a series of changes driving BM-MSCs cultured in a monolayer towards chondrocyte-like cells 7 days after treatment. No changes were observed in intact (nonpermeabilized) BM-MSCs incubated in chondrocyte extract. These results are consistent with those of Taranger et al (19) and Bru et al (17), but not with those by Hansis et al (20). However, no changes were detected on day 14. In addition 
to this finding, the percentage of permeabilized cells in this study was over $55 \%$ and was discrepant with COL II-positive cells. There are various reasons for this finding: i) A low concentration of cell extract leads to incomplete transdifferentiation; ii) preferential growth of BM-MSCs inhibits the proliferation of successfully reprogrammed BM-MSCs; iii) the culturing method makes the chondrogenic microenvironment deficient and iv) the changes were transient and could not be detected at the 14th day.

Considering the fact that chondroadherin interaction with cells may be crucial for maintaining the adult chondrocyte phenotype and cartilage homeostasis, cell-cell contact may be responsible for the reprogramming (21). Fourteen days after micromass culture, the cells presented a crumb structure. $\mathrm{H} \& \mathrm{E}$ staining showed cells in the crumb presenting lacunae structure with chondroitin sulfate and were COL II-positive. This suggests that cytokines secreted by BM-MSC-converted chondrocytes are likely to constantly induce BM-MSCs towards chondrocytes.

The main regulatory component involved in the cell extract was believed to initiate a transcriptional program specific for the alteration (3). Hakelien et al (2) observed the import of transcription factors into 293T nuclei in their study. At the same time, demethylation of the target cell-specific gene promoter and histone acetylation were also observed $(3,5,13,17)$.

Cell reprogramming is of interest for the development of novel cellular therapeutics and is potentially useful for producing isogenic replacement cells for the treatment of a wide variety of diseases. The incomplete transcriptional and epigenetic reprogramming may not lead the cells harvested in this manner to be totally the same as the 'target' cells, but own some of the desired functions of the 'target' cells. To a certain degree, it is sufficient for cell programming to exhibit the necessary therapeutic properties without complete alteration of genes, protein and functions. For example, rat fibroblasts reversibly permeabilized with SLO and exposed to extract of an insulin-producing $\beta$ cell line were capable of secreting insulin on day 5 (3). For diabetes care, it is sufficient to harvest cells able to secrete insulin.

Since only this alteration is detectable, it indicates a limitation of our study. Thus, the relevant mechanism involved in this process and the methods of increasing efficiency and the maintenance time of reprogramming should be investigated.

In conclusion, this study has shown that reversibly permeabilized BM-MSCs were differentiated into chondrocytes when they were co-cultured with the chondrocyte extract. SLO plays a key role, but not dependently, in the reprogramming process and it sets the stage for the further action of more essential components in the cell extract. Nevertheless, culturing cells with a high-density protocol is likely to prolong the phenotypic changes of differentiated cells. The method used in this study provides tissue engineering with a novel way of obtaining seeding cells.

\section{Acknowledgements}

This study was supported by the National Natural Science Foundation of China (Nos. 30900308 and 81071580).

\section{References}

1. Wong CC, Chiu LH, Lai WF, et al: Phenotypic re-expression of near quiescent chondrocytes: The effects of type II collagen and growth factors. J Biomater Appl 25: 75-95, 2010.

2. Hakelien AM, Landsverk HB, Robl JM, Skalhegg BS and Collas P: Reprogramming fibroblasts to express T-cell functions using cell extracts. Nat Biotechnol 20: 460-466, 2002.

3. Hakelien AM, Gaustad KG and Collas P: Transient alteration of cell fate using a nuclear and cytoplasmic extract of an insulinoma cell line. Biochem Biophys Res Commun 316: 834-841, 2004.

4. Gaustad KG, Boquest AC, Anderson BE, Gerdes AM and Collas P: Differentiation of human adipose tissue stem cells using extracts of rat cardiomyocytes. Biochem Biophys Res Commun 314: 420-427, 2004

5. Miyamoto K, Furusawa T, Ohnuki M, et al: Reprogramming events of mammalian somatic cells induced by Xenopus laevis egg extracts. Mol Reprod Dev 74: 1268-1277, 2007.

6. Wu Y, Chen L, Scott PG and Tredget EE: Mesenchymal stem cells enhance wound healing through differentiation and angiogenesis. Stem Cells 25: 2648-2659, 2007.

7. Li H, Zan T, Li Y, et al: Transplantation of adipose-derived stem cells promotes formation of prefabricated flap in a rat model. Tohoku J Exp Med 222: 131-140, 2010.

8. Pittenger MF, Mackay AM, Beck SC, et al: Multilineage potential of adult human mesenchymal stem cells. Science 284 : 143-147, 1999.

9. Indrawattana N, Chen G, Tadokoro M, et al: Growth factor combination for chondrogenic induction from human mesenchymal stem cell. Biochem Biophys Res Commun 320: 914-919, 2004.

10. Thompson AD, Betz MW, Yoon DM and Fisher JP: Osteogenic differentiation of bone marrow stromal cells induced by coculture with chondrocytes encapsulated in three-dimensional matrices. Tissue Eng Part A 15: 1181-1190, 2009.

11. Liu X, Sun H, Yan D, et al: In vivo ectopic chondrogenesis of BMSCs directed by mature chondrocytes. Biomaterials 31: 9406-9414, 2010.

12. Lee HJ, Choi BH, Min BH, Son YS and Park SR: Low-intensity ultrasound stimulation enhances chondrogenic differentiation in alginate culture of mesenchymal stem cells. Artif Organs 30: 707-715, 2006

13. Rajasingh J, Lambers E, Hamada $\mathrm{H}$, et al: Cell-free embryonic stem cell extract-mediated derivation of multipotent stem cells from NIH3T3 fibroblasts for functional and anatomical ischemic tissue repair. Circ Res 102: e107-e117, 2008.

14. Walev I, Bhakdi SC, Hofmann F, et al: Delivery of proteins into living cells by reversible membrane permeabilization with streptolysin-O. Proc Natl Acad Sci USA 98: 3185-3190, 2001.

15. Palmer M, Vulicevic I, Saweljew P, Valeva A, Kehoe M and Bhakdi S: Streptolysin O: a proposed model of allosteric interaction between a pore-forming protein and its target lipid bilayer. Biochemistry 37: 2378-2383, 1998.

16. Palmer M, Harris R, Freytag C, Kehoe M, Tranum-Jensen J and Bhakdi S: Assembly mechanism of the oligomeric streptolysin $\mathrm{O}$ pore: the early membrane lesion is lined by a free edge of the lipid membrane and is extended gradually during oligomerization. EMBO J 17: 1598-1605, 1998.

17. Bru T, Clarke C, McGrew MJ, Sang HM, Wilmut I and Blow JJ: Rapid induction of pluripotency genes after exposure of human somatic cells to mouse ES cell extracts. Exp Cell Res 314: 2634-2642, 2008.

18. Miyamoto K, Tsukiyama T, Yang Y, et al: Cell-free extracts from mammalian oocytes partially induce nuclear reprogramming in somatic cells. Biol Reprod 80: 935-943, 2009.

19. Taranger CK, Noer A, Sorensen AL, Hakelien AM, Boquest AC and Collas P: Induction of dedifferentiation, genomewide transcriptional programming, and epigenetic reprogramming by extracts of carcinoma and embryonic stem cells. Mol Biol Cell 16: 5719-5735, 2005.

20. Hansis C, Barreto G, Maltry N and Niehrs C: Nuclear reprogramming of human somatic cells by Xenopus egg extract requires BRG1. Curr Biol 14: 1475-1480, 2004.

21. Yin S, Cen L, Wang C, et al: Chondrogenic transdifferentiation of human dermal fibroblasts stimulated with cartilage-derived morphogenetic protein 1. Tissue Eng Part A 16: 1633-1643, 2010. 